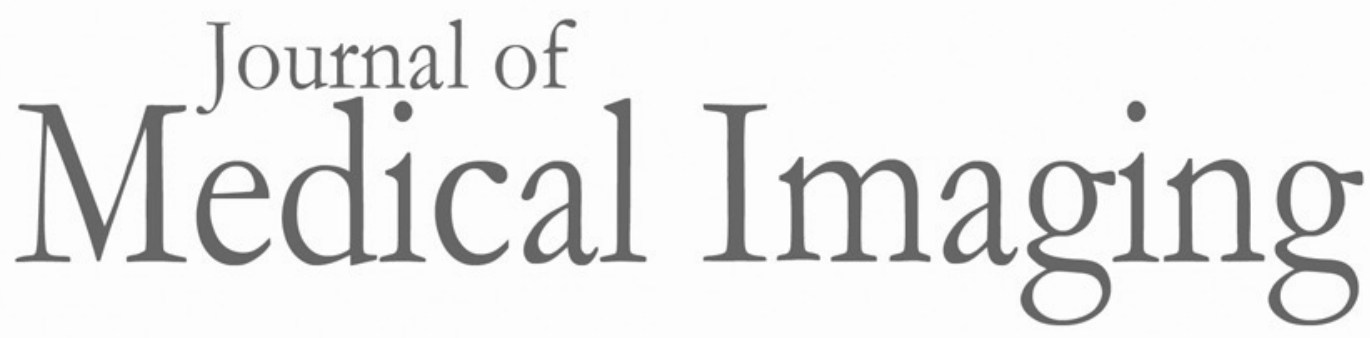

\title{
Errata: Spectral response model for a multibin photon-counting spectral computed tomography detector and its applications
}

Xuejin Liu

Mats Persson

Hans Bornefalk

Staffan Karlsson

Cheng Xu

Mats Danielsson

Ben Huber 


\section{Errata: Spectral response model for a multibin photon-counting spectral computed tomography detector and its applications}

Xuejin Liu, Mats Persson, Hans Bornefalk, Staffan Karlsson, Cheng Xu, Mats Danielsson, and Ben Huber KTH Royal Institute of Technology, Department of Physics, Roslagstullsbacken 21, Stockholm 10691, Sweden

[DOI: 10.1117/1.JMI.3.4.049801]

This article [J. Med. Imag. 2(3), 033502 (2015)] was originally published online on 11 September 2015 with an error on p. 3. At the bottom of the left column, the formula $\sigma=135 \mathrm{keV}$ has been replaced by $\sigma=1.35 \mathrm{keV}$.
On p. 8, Fig. 8 is wrong due to the miscalculated Hounsfield unit for these bin images. The correct figure is reproduced below.

This article was corrected online on 14 October 2016.
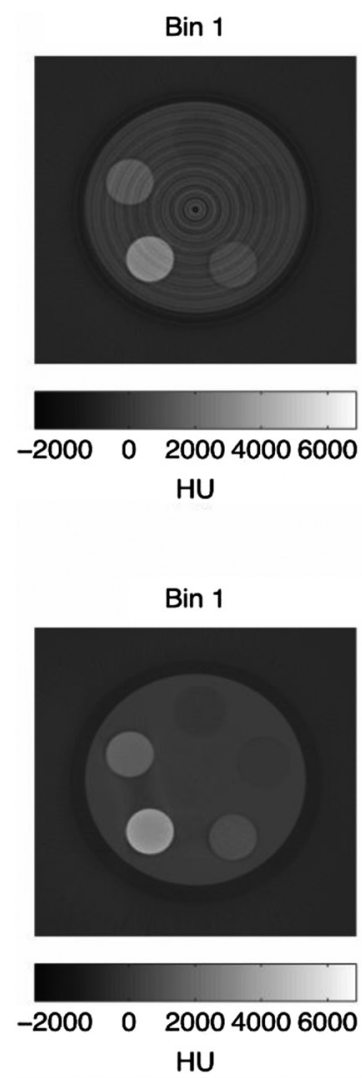

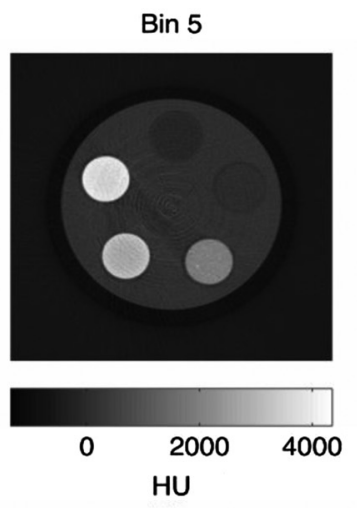

(a)

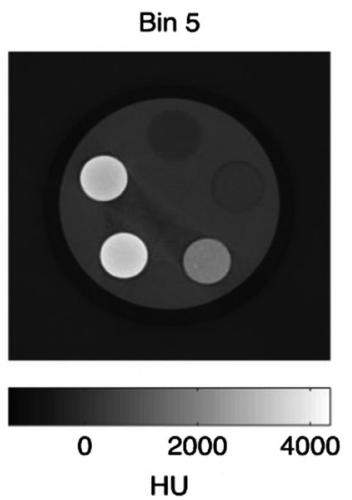

(b)
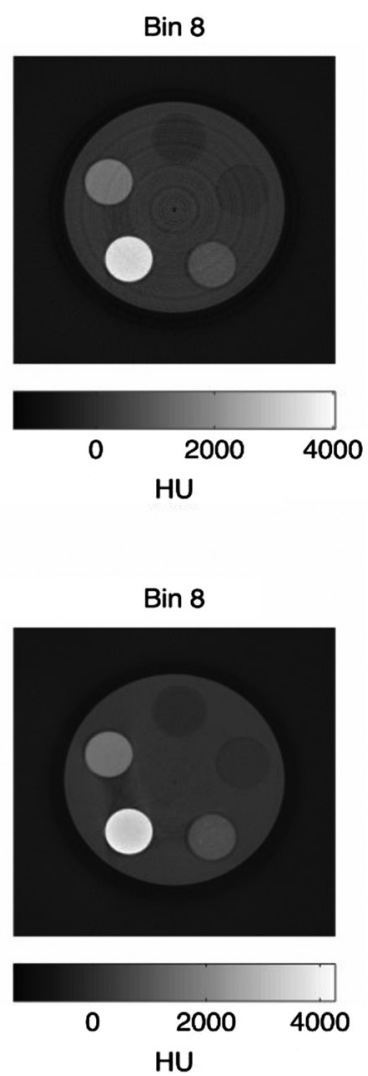

HU

Fig. 8 Reconstructed bin images of energy bin 1, bin 5, and bin 8. (a) are the bin images reconstructed from the raw projection data that are normalized by flat fielding using an air scan. (b) are the bin images reconstructed from the projection data that are processed with the inhomogeneity compensation method. All images are normalized to HU measured in the water insert. 